\title{
A discrepancy principle for equations with monotone continuous operators
}

\author{
N.S. Hoang, A.G. Ramm* \\ Mathematics Department, Kansas State University, Manhattan, KS 66506-2602, USA
}

\section{A R T I C L E I N F O}

\section{Article history:}

Received 18 September 2008

Accepted 30 September 2008

\section{MSC:}

47J05

47J06

$47 \mathrm{~J} 35$

$65 \mathrm{R} 30$

\section{Keywords:}

\section{Discrepancy principle}

Monotone operators

Regularization

Nonlinear operator equations

Ill-posed problems

\begin{abstract}
A B S T R A C T
A discrepancy principle for solving nonlinear equations with monotone operators given noisy data is formulated. The existence and uniqueness of the corresponding regularization parameter $a(\delta)$ are proved. Convergence of the solution obtained by the discrepancy principle is justified. The results are obtained under natural assumptions on the nonlinear operator.
\end{abstract}

(c) 2008 Elsevier Ltd. All rights reserved.

\section{Introduction}

Consider the equation:

$$
F(u)=f,
$$

where $F$ is a monotone operator in a real Hilbert space $H$. Monotonicity is understood in the following sense:

$$
\langle F(u)-F(v), u-v\rangle \geq 0, \quad \forall u, v \in H .
$$

Here $\langle\cdot, \cdot\rangle$ denotes the inner product in $H$. Assume that $F$ is continuous.

Equations with monotone operators are important in many applications and were studied extensively, see, for example, $[1-3,9,10,12]$, and references therein. There are many technical and physical problems leading to equations with such operators in the cases when dissipation of energy occurs. For example, in [5,4], Chapter 3, pp. 156-189, a wide class of nonlinear dissipative systems is studied, and the basic equations of such systems can be reduced to (1) with monotone operators. Many examples of equations with monotone operators can be found in [2] and in references mentioned above. In [6,7] it is proved that any solvable linear operator equation with a closed densely defined operator in a Hilbert space $H$ can be reduced to an equation with a monotone operator and solved by a convergent iterative process.

In this paper, apparently for the first time, a discrepancy principle for solving Eq. (3) with noisy data (see Section 2) is proved under natural assumptions. No smallness assumptions on the nonlinearity, no global restrictions on its growth, or other special properties of the nonlinearity, except the monotonicity and continuity, are imposed. No source-type

\footnotetext{
* Corresponding author.

E-mail addresses: nguyenhs@math.ksu.edu (N.S. Hoang), ramm@math.ksu.edu (A.G. Ramm).
} 
assumptions are used. Our result is widely applicable. It is well known that without extra assumptions, usually source-type assumption concerning the right-hand side, or some equivalent assumption concerning the smoothness of the solution, one cannot get a rate of convergence even for linear ill-posed equations (see, for example, [9]). On the other hand, such assumptions are usually not algorithmically verifiable and often they do not hold. For this reason we do not make such assumptions and do not give estimates of the rate of convergence.

In [11] a stationary equation $F(u)=f$ with a nonlinear monotone operator $F$ was studied. Assumptions A1-A3 on p.197 in [11] are more restrictive than ours, and the Rule R2 on p.199, formula (4.1) in [11], for the choice of the regularization parameter is more difficult to use computationally: one has to solve nonlinear Equation 4.1 in [11] for the regularization parameter. Moreover, to use this equation one has to invert an ill-conditioned linear operator $A+a I$ for small values of $a$. Assumption A1 in [11] is not verifiable, because the solution $x^{\dagger}$ is not known. Assumption A3 in [11] requires $F$ to be constant in a ball $B_{r}\left(x^{\dagger}\right)$ if $F^{\prime}\left(x^{\dagger}\right)=0$. Our discrepancy principle does not require these assumptions, and, in contrast to Equation 4.1 in [11], it does not require inversion of ill-conditioned linear operators.

The novel results in our paper include Theorem 5 in Section 3 and Theorem 7 in Section 4. In Theorem 5 a new discrepancy principle is proposed and justified assuming only the monotonicity and continuity of $F$. Implementing the discrepancy principle in Theorem 5 requires solving Eq. (3) and then solving nonlinear equation (15) for the regularization parameter $a(\delta)$. Theorem 7 allows one to solve Eqs. (3) and (15) approximately. Thus, when $\delta$ is not too small one can save a large amount of computation in solving Eqs. (3) and (15) by applying Theorem 7 and using our new stopping rule. Our results allow one to solve numerically stably equation (1) if $F$ is locally Lipschitz and monotone. Based on Theorem 7, an algorithm for stable solution of Eq. (1) is formulated for locally Lipschitz monotone operators.

\section{Auxiliary results}

Let us consider the following equation

$$
F\left(V_{\delta, a}\right)+a V_{\delta, a}-f_{\delta}=0, \quad a>0,
$$

where $a=$ const. It is known (see, e.g., [9, p.111]) that Eq. (3) with monotone continuous operator $F$ has a unique solution for any $f_{\delta} \in H$.

Throughout the paper we assume that $F$ is a monotone continuous operator and the inner product in $H$ is denoted $\langle u, v\rangle$. Below the word decreasing means strictly decreasing and increasing means strictly increasing.

Recall the following result from [9, p.112]:

Lemma 1. Assume that Eq. (1) is solvable, $y$ is its minimal-norm solution, assumption (2) holds, and $F$ is continuous. Then

$$
\lim _{a \rightarrow 0}\left\|V_{a}-y\right\|=0,
$$

where $V_{a}$ solves Eq. (3) with $\delta=0$.

Lemma 2. Assume $\left\|F(0)-f_{\delta}\right\|>0$. Let $a>0$, and $F$ be monotone. Denote

$$
\psi(a):=\left\|V_{\delta, a}\right\|, \quad \phi(a):=a \psi(a)=\left\|F\left(V_{\delta, a}\right)-f_{\delta}\right\|,
$$

where $V_{\delta, a}$ solves (3). Then $\psi(a)$ is decreasing, and $\phi(a)$ is increasing.

Proof. Since $\left\|F(0)-f_{\delta}\right\|>0$, one has $\psi(a) \neq 0, \forall a \geq 0$. Indeed, if $\left.\psi(a)\right|_{a=\tau}=0$, then $V_{\delta, a}=0$, and Eq. (3) implies $\left\|F(0)-f_{\delta}\right\|=0$, which is a contradiction. Note that $\phi(a)=a\left\|V_{\delta, a}\right\|$. One has

$$
\begin{aligned}
0 & \leq\left\langle F\left(V_{\delta, a}\right)-F\left(V_{\delta, b}\right), V_{\delta, a}-V_{\delta, b}\right\rangle \\
& =\left\langle-a V_{\delta, a}+b V_{\delta, b}, V_{\delta, a}-V_{\delta, b}\right\rangle \\
& =(a+b)\left\langle V_{\delta, a}, V_{\delta, b}\right\rangle-a\left\|V_{\delta, a}\right\|^{2}-b\left\|V_{\delta, b}\right\|^{2} .
\end{aligned}
$$

Thus,

$$
\begin{aligned}
0 & \leq(a+b)\left\langle V_{\delta, a}, V_{\delta, b}\right\rangle-a\left\|V_{\delta, a}\right\|^{2}-b\left\|V_{\delta, b}\right\|^{2} \\
& \leq(a+b)\left\|V_{\delta, a}\right\|\left\|V_{\delta, b}\right\|-a\left\|V_{\delta, a}\right\|^{2}-b\left\|V_{\delta, b}\right\|^{2} \\
& =\left(a\left\|V_{\delta, a}\right\|-b\left\|V_{\delta, b}\right\|\right)\left(\left\|V_{\delta, b}\right\|-\left\|V_{\delta, a}\right\|\right) \\
& =(\phi(a)-\phi(b))(\psi(a)-\psi(b)) .
\end{aligned}
$$

If $\psi(b)>\psi(a)$ then (6) implies $\phi(a) \geq \phi(b)$, so

$$
a \psi(a) \geq b \psi(b)>b \psi(a) .
$$

Therefore, if $\psi(b)>\psi(a)$ then $b<a$.

Similarly, if $\psi(b)<\psi(a)$ then $\phi(a) \leq \phi(b)$. This implies $b>a$. 
Suppose $\psi(a)=\psi(b)$, i.e., $\left\|V_{\delta, a}\right\|=\left\|V_{\delta, b}\right\|$. From (5) one has

$$
\left\|V_{\delta, a}\right\|^{2} \leq\left\langle V_{\delta, a}, V_{\delta, b}\right\rangle \leq\left\|V_{\delta, a}\right\|\left\|V_{\delta, b}\right\|=\left\|V_{\delta, a}\right\|^{2} .
$$

This implies $V_{\delta, a}=V_{\delta, b}$, and then Eq. (3) implies $a=b$.

Therefore $\phi$ is increasing and $\psi$ is decreasing.

Lemma 3. If $F$ is monotone and continuous, then $\left\|V_{\delta, a}\right\|=O\left(\frac{1}{a}\right)$ as $a \rightarrow \infty$, and

$$
\lim _{a \rightarrow \infty}\left\|F\left(V_{\delta, a}\right)-f_{\delta}\right\|=\left\|F(0)-f_{\delta}\right\|
$$

Proof. Rewrite (3) as

$$
F\left(V_{\delta, a}\right)-F(0)+a V_{\delta, a}+F(0)-f_{\delta}=0 .
$$

Multiply this equation by $V_{\delta, a}$, use the monotonicity of $F$ and get:

$$
a\left\|V_{\delta, a}\right\|^{2} \leq\left\langle a V_{\delta, a}+F\left(V_{\delta, a}\right)-F(0), V_{\delta, a}\right\rangle=\left\langle f_{\delta}-F(0), V_{\delta, a}\right\rangle \leq\left\|f_{\delta}-F(0)\right\|\left\|V_{\delta, a}\right\| .
$$

Therefore, $\left\|V_{\delta, a}\right\|=O\left(\frac{1}{a}\right)$. This and the continuity of $F$ imply (7).

Remark 1. If $\left\|F(0)-f_{\delta}\right\|>C \delta^{\gamma}, 0<\gamma \leq 1$ then relation (7) implies

$$
\left\|F\left(V_{\delta, a}\right)-f_{\delta}\right\| \geq C \delta^{\gamma}, \quad 0<\gamma \leq 1,
$$

for sufficiently large $a>0$.

Lemma 4. Let $C>0$ and $\gamma \in(0,1]$ be constants such that $C \delta^{\gamma}>\delta$. Suppose that $\left\|F(0)-f_{\delta}\right\|>C \delta^{\gamma}$. Then, there exists a unique $a(\delta)>0$ such that $\left\|F\left(V_{\delta, a(\delta)}\right)-f_{\delta}\right\|=C \delta^{\gamma}$.

Proof. We have $F(y)=f$, and

$$
\begin{aligned}
0 & =\left\langle F\left(V_{\delta, a}\right)+a V_{\delta, a}-f_{\delta}, F\left(V_{\delta, a}\right)-f_{\delta}\right\rangle \\
& =\left\|F\left(V_{\delta, a}\right)-f_{\delta}\right\|^{2}+a\left\langle V_{\delta, a}-y, F\left(V_{\delta, a}\right)-f_{\delta}\right\rangle+a\left\langle y, F\left(V_{\delta, a}\right)-f_{\delta}\right\rangle \\
& =\left\|F\left(V_{\delta, a}\right)-f_{\delta}\right\|^{2}+a\left\langle V_{\delta, a}-y, F\left(V_{\delta, a}\right)-F(y)\right\rangle+a\left\langle V_{\delta, a}-y, f-f_{\delta}\right\rangle+a\left\langle y, F\left(V_{\delta, a}\right)-f_{\delta}\right\rangle \\
& \geq\left\|F\left(V_{\delta, a}\right)-f_{\delta}\right\|^{2}+a\left\langle V_{\delta, a}-y, f-f_{\delta}\right\rangle+a\left\langle y, F\left(V_{\delta, a}\right)-f_{\delta}\right\rangle .
\end{aligned}
$$

Here the monotonicity of $F$ was used. Therefore

$$
\begin{aligned}
\left\|F\left(V_{\delta, a}\right)-f_{\delta}\right\|^{2} & \leq-a\left\langle V_{\delta, a}-y, f-f_{\delta}\right\rangle-a\left\langle y, F\left(V_{\delta, a}\right)-f_{\delta}\right\rangle \\
& \leq a\left\|V_{\delta, a}-y\right\|\left\|f-f_{\delta}\right\|+a\|y\|\left\|F\left(V_{\delta, a}\right)-f_{\delta}\right\| \\
& \leq a \delta\left\|V_{\delta, a}-y\right\|+a\|y\|\left\|F\left(V_{\delta, a}\right)-f_{\delta}\right\| .
\end{aligned}
$$

Also,

$$
\begin{aligned}
0 & =\left\langle F\left(V_{\delta, a}\right)-F(y)+a V_{\delta, a}+f-f_{\delta}, V_{\delta, a}-y\right\rangle \\
& =\left\langle F\left(V_{\delta, a}\right)-F(y), V_{\delta, a}-y\right\rangle+a\left\|V_{\delta, a}-y\right\|^{2}+a\left\langle y, V_{\delta, a}-y\right\rangle+\left\langle f-f_{\delta}, V_{\delta, a}-y\right\rangle \\
& \geq a\left\|V_{\delta, a}-y\right\|^{2}+a\left\langle y, V_{\delta, a}-y\right\rangle+\left\langle f-f_{\delta}, V_{\delta, a}-y\right\rangle,
\end{aligned}
$$

where the monotonicity of $F$ was used again. Therefore,

$$
a\left\|V_{\delta, a}-y\right\|^{2} \leq a\|y\|\left\|V_{\delta, a}-y\right\|+\delta\left\|V_{\delta, a}-y\right\| .
$$

This implies

$$
a\left\|V_{\delta, a}-y\right\| \leq a\|y\|+\delta .
$$

From (9), (10), and an elementary inequality $a b \leq \epsilon a^{2}+\frac{b^{2}}{4 \epsilon}, \forall \epsilon>0$, one gets:

$$
\begin{aligned}
\left\|F\left(V_{\delta, a}\right)-f_{\delta}\right\|^{2} & \leq \delta^{2}+a\|y\| \delta+a\|y\|\left\|F\left(V_{\delta, a}\right)-f_{\delta}\right\| \\
& \leq \delta^{2}+a\|y\| \delta+\epsilon\left\|F\left(V_{\delta, a}\right)-f_{\delta}\right\|^{2}+\frac{1}{4 \epsilon} a^{2}\|y\|^{2},
\end{aligned}
$$

where $\epsilon>0$ is arbitrary small, fixed, independent of $a$, and can be chosen arbitrary small. Let $a \searrow 0$. Then (11) implies $\lim _{a \rightarrow 0}(1-\epsilon)\left\|F\left(V_{\delta, a}\right)-f_{\delta}\right\|^{2} \leq \delta^{2}<\left(C \delta^{\gamma}\right)^{2}$. Thus,

$$
\lim _{a \rightarrow 0}\left\|F\left(V_{\delta, a}\right)-f_{\delta}\right\|<C \delta^{\gamma}, \quad C>0,0<\gamma \leq 1 .
$$


This, the continuity of $F$, the continuity of $V_{\delta, a}$ with respect to $a \in[0, \infty)$, and inequality (8), imply that equation $\left\|F\left(V_{\delta, a}\right)-f_{\delta}\right\|=C \delta^{\gamma}$ must have a solution $a(\delta)>0$.

Remark 2. Let $V_{a}:=\left.V_{\delta, a}\right|_{\delta=0}$, so $F\left(V_{a}\right)+a V-f=0$. Let $y$ be the minimal-norm solution to Eq. (1). We claim that

$$
\left\|V_{\delta, a}-V_{a}\right\| \leq \frac{\delta}{a}
$$

Indeed, from (3) one gets

$$
F\left(V_{\delta, a}\right)-F\left(V_{a}\right)+a\left(V_{\delta, a}-V_{a}\right)=f-f_{\delta} .
$$

Multiply this equality by $\left(V_{\delta, a}-V_{a}\right)$ and use (2) to obtain

$$
\begin{aligned}
\delta\left\|V_{\delta, a}-V_{a}\right\| & \geq\left\langle f-f_{\delta}, V_{\delta, a}-V_{a}\right\rangle \\
& =\left\langle F\left(V_{\delta, a}\right)-F\left(V_{a}\right)+a\left(V_{\delta, a}-V_{a}\right), V_{\delta, a}-V_{a}\right\rangle \\
& \geq a\left\|V_{\delta, a}-V_{a}\right\|^{2} .
\end{aligned}
$$

This implies (12).

Let us derive a uniform with respect to $a$ bound on $\left\|V_{a}\right\|$. From the equation

$$
F\left(V_{a}\right)+a V_{a}-F(y)=0,
$$

and the monotonicity of $F$ one gets

$$
0=\left\langle F\left(V_{a}\right)+a V_{a}-F(y), V_{a}-y\right\rangle \geq a\left\langle V_{a}, V_{a}-y\right\rangle .
$$

This implies the desired bound:

$$
\left\|V_{a}\right\| \leq\|y\|, \quad \forall a>0 .
$$

Similar arguments can be found in [9, p. 113].

From (12) and (13), one gets the following estimate:

$$
\left\|V_{\delta, a}\right\| \leq\left\|V_{a}\right\|+\frac{\delta}{a} \leq\|y\|+\frac{\delta}{a} .
$$

\section{A discrepancy principle}

Our standing assumptions are the monotonicity and continuity of $F$ and the solvability of Eq. (1). They are not repeated below. We assume without loss of generality that $\delta \in(0,1)$.

Theorem 5. Let $\gamma \in(0,1]$ and $C>0$ be some constants such that $C \delta^{\gamma}>\delta$. Assume that $\left\|F(0)-f_{\delta}\right\|>C \delta^{\gamma}$. Let $y$ be its minimal-norm solution. Then there exists a unique $a(\delta)>0$ such that

$$
\left\|F\left(V_{\delta, a(\delta)}\right)-f_{\delta}\right\|=C \delta^{\gamma},
$$

where $V_{\delta, a(\delta)}$ solves (3) with $a=a(\delta)$.

If $0<\gamma<1$ then

$$
\lim _{\delta \rightarrow 0}\left\|V_{\delta, a(\delta)}-y\right\|=0 .
$$

Proof. The existence and uniqueness of $a(\delta)$ follow from Lemma 4. Let us show that

$$
\lim _{\delta \rightarrow 0} a(\delta)=0 .
$$

The triangle inequality, inequality (12) and equality (15) imply

$$
\begin{aligned}
a(\delta)\left\|V_{a(\delta)}\right\| & \leq a(\delta)\left(\left\|V_{\delta, a(\delta)}-V_{a(\delta)}\right\|+\left\|V_{\delta, a(\delta)}\right\|\right) \\
& \leq \delta+a(\delta)\left\|V_{\delta, a(\delta)}\right\|=\delta+C \delta^{\gamma} .
\end{aligned}
$$

From inequality (18), one gets

$$
\lim _{\delta \rightarrow 0} a(\delta)\left\|V_{a(\delta)}\right\|=0 .
$$

It follows from Lemma 2 with $f_{\delta}=f$, i.e., $\delta=0$, that the function $\phi_{0}(a):=a\left\|V_{a}\right\|$ is nonnegative and strictly increasing on $(0, \infty)$. This and relation (19) imply:

$$
\lim _{\delta \rightarrow 0} a(\delta)=0 .
$$


From (15) and (14), one gets

$$
C \delta^{\gamma}=a\left\|V_{\delta, a}\right\| \leq a(\delta)\|y\|+\delta .
$$

Thus, one gets:

$$
C \delta^{\gamma}-\delta \leq a(\delta)\|y\| .
$$

If $\gamma<1$ then $C-\delta^{1-\gamma}>0$ for sufficiently small $\delta$. This implies:

$$
0 \leq \lim _{\delta \rightarrow 0} \frac{\delta}{a(\delta)} \leq \lim _{\delta \rightarrow 0} \frac{\delta^{1-\gamma}\|y\|}{C-\delta^{1-\gamma}}=0 .
$$

By the triangle inequality and inequality (12), one has

$$
\left\|V_{\delta, a(\delta)}-y\right\| \leq\left\|V_{a(\delta)}-y\right\|+\left\|V_{a(\delta)}-V_{\delta, a(\delta)}\right\| \leq\left\|V_{a(\delta)}-y\right\|+\frac{\delta}{a(\delta)} .
$$

Relation (16) follows from (23), (24) and Lemma 1.

Instead of using (3), one may use the following equation:

$$
F\left(V_{\delta, a}\right)+a\left(V_{\delta, a}-\bar{u}\right)-f_{\delta}=0, \quad a>0,
$$

where $\bar{u}$ is an element of $H$. Denote $F_{1}(u):=F(u+\bar{u})$. Then $F_{1}$ is monotone and continuous. Eq. (3) can be written as:

$$
F_{1}\left(U_{\delta, a}\right)+a U_{\delta, a}-f_{\delta}=0, \quad U_{\delta, a}:=V_{\delta, a}-\bar{u}, a>0 .
$$

By applying Theorem 5 with $F=F_{1}$ one gets the following result:

Corollary 6. Let $\gamma \in(0,1]$ and $C>0$ be some constants such that $C \delta^{\gamma}>\delta$. Let $\bar{u} \in H$ and $z$ be the solution to (1) with minimal distance to $\bar{u}$. Assume that $\left\|F(\bar{u})-f_{\delta}\right\|>C \delta^{\gamma}$. Then there exists a unique $a(\delta)>0$ such that

$$
\left\|F\left(\tilde{V}_{\delta, a(\delta)}\right)-f_{\delta}\right\|=C \delta^{\gamma},
$$

where $\tilde{V}_{\delta, a(\delta)}$ solves the following equation:

$$
F\left(\tilde{V}_{\delta, a}\right)+a(\delta)\left(\tilde{V}_{\delta, a}-\bar{u}\right)-f_{\delta}=0 .
$$

If $\gamma \in(0,1)$ then this $a(\delta)$ satisfies

$$
\lim _{\delta \rightarrow 0}\left\|\tilde{V}_{\delta, a(\delta)}-z\right\|=0 .
$$

Remark 3. It is an open problem to choose $\gamma$ and $C$ optimal in some sense.

Remark 4. Theorems 5 and 7 do not hold, in general, for $\gamma=1$. Indeed, let $F u=\langle u, p\rangle p,\|p=1\|, p \perp \mathcal{N}(F):=\{u \in$ $H: F u=0\}, f=p, f_{\delta}=p+q \delta$, where $\langle p, q\rangle=0,\|q\|=1, F q=0,\|q \delta\|=\delta$. One has $F y=p$, where $y=p$, is the minimal-norm solution to the equation $F u=p$. Equation $F u+a u=p+q \delta$, has the unique solution $V_{\delta, a}=q \delta / a+p /(1+a)$. Eq. (15) is $C \delta=\|q \delta+(a p) /(1+a)\|$. This equation yields $a=a(\delta)=c \delta /(1-c \delta)$, where $c:=\left(C^{2}-1\right)^{1 / 2}$, and we assume $c \delta<1$. Thus, $\lim _{\delta \rightarrow 0} V_{\delta, a(\delta)}=p+c^{-1} q:=v$, and $F v=p$. Therefore $v=\lim _{\delta \rightarrow 0} V_{\delta, a(\delta)}$ is not $p$, i.e., is not the minimal-norm solution to the equation $F u=p$. Similar arguments can be found in [8, p. 29].

\section{Applications}

In this section we discuss methods for solving Eqs. (1) and (3) using the new discrepancy principle, i.e., Theorem 5. Implementing this principle, i.e., solving Eq. (15), requires solving Eq. (3). If $F$ is linear, then Eq. (3) has the form:

$$
(F+a I) u=f_{\delta} .
$$

Since $F \geq 0$ the operator $F+a I$ is boundedly invertible, $\left\|(F+a I)^{-1}\right\| \leq \frac{1}{a}$, and Eq. (29) is well-posed if $a>0$ is not too small. There are many methods for solving efficiently well-posed linear equations with positive-definite operators. For this reason we mainly discuss some methods for a stable solution of Eq. (1) with nonlinear operators. In this section a method is developed for a stable solution of Eq. (1) with locally Lipschitz monotone operator $F$, so we assume that

$$
\|F(u)-F(v)\| \leq L\|u-v\|, \quad u, v \in B\left(u_{0}, R\right):=\left\{u:\left\|u-u_{0}\right\| \leq R\right\}, \quad L=L(R) .
$$

Here $u_{0} \in H$ is an arbitrary fixed element. Consider the operator

$$
G(u):=u-\lambda\left[F(u)+a u-f_{\delta}\right], \quad \lambda>0 .
$$


We claim that $G$ is a contraction mapping in $H$ provided that $\lambda$ is sufficiently small. Let $F_{1}:=F+a I$. Then (30) implies $\left\|F_{1}(u)-F_{1}(v)\right\| \leq(a+L)\|u-v\|$. Using the monotonicity of $F$, one gets

$$
\begin{aligned}
\|G(u)-G(v)\|^{2} & =\left\|(u-v)-\lambda\left(F_{1}(u)-F_{1}(v)\right)\right\|^{2} \\
& =\|u-v\|^{2}-2 \lambda\left\langle u-v, F_{1}(u)-F_{1}(v)\right\rangle+\lambda^{2}\left\|F_{1}(u)-F_{1}(v)\right\|^{2} \\
& \leq\|u-v\|^{2}\left[1-2 \lambda a+\lambda^{2}(a+L)^{2}\right] .
\end{aligned}
$$

This implies that $G$ is a contraction mapping if

$$
0<\lambda<\frac{2 a}{(a+L)^{2}} .
$$

For these $\lambda$ the solution $V_{\delta, a}$ of Eq. (3) can be found by the following iterative process:

$$
u_{n+1}=u_{n}-\lambda\left[F\left(u_{n}\right)+a u_{n}-f_{\delta}\right], \quad u_{0}:=u_{0} .
$$

After finding $V_{\delta, a}$, one finds $a(\delta)$ from the discrepancy principle (15), i.e., by solving the nonlinear equation:

$$
\phi(a(\delta)):=\left\|F\left(V_{\delta, a(\delta)}\right)-f_{\delta}\right\|=C \delta^{\gamma} .
$$

There are many methods for solving this equation. For example, one can use the bisection method or the golden section method. If $a(\delta)$ is found, one solves Eq. (3) with $a=a(\delta)$ for $V_{\delta, a(\delta)}$ and takes its solution as an approximate solution to (1).

Although the sequence $u_{n}$, defined by (32), converges to the solution of Eq. (3) at the rate of a geometrical series with a denominator $q \in(0,1)$, it is very time consuming to try to solve Eq. (3) with high accuracy if $q$ is close to 1 . Theorem 7 (see below) allows one to stop iterations (32) at the first value of $n$ which satisfies the following condition:

$$
\left\|F\left(u_{n}\right)+a u_{n}-f_{\delta}\right\| \leq \theta \delta, \quad \theta>0,
$$

where $\theta$ is a fixed constant. This saves the time of computation.

Theorem 7. Let $\delta, F, f_{\delta}$, and $y$ be as in Theorem 5 and $0<\gamma<1$. Assume that $v_{\delta} \in H$ and $\alpha(\delta)>0$ satisfy the following conditions:

$$
\left\|F\left(v_{\delta}\right)+\alpha(\delta) v_{\delta}-f_{\delta}\right\| \leq \theta \delta,
$$

and

$$
C_{1} \delta^{\gamma} \leq\left\|F\left(v_{\delta}\right)-f_{\delta}\right\| \leq C_{2} \delta^{\gamma}, \quad 0<C_{1}<C_{2} .
$$

Then one has:

$$
\lim _{\delta \rightarrow 0}\left\|v_{\delta}-y\right\|=0 .
$$

Proof. Let $u$ and $v$ be arbitrary elements in $H$. By the monotonicity of $F$ one gets

$$
\begin{aligned}
a\|u-v\|^{2} & \leq\langle u-v, F(u)-F(v)+a u-a v\rangle \\
& \leq\|u-v\|\|F(u)-F(v)+a u-a v\|, \quad \forall a>0 .
\end{aligned}
$$

This implies

$$
a\|u-v\| \leq\|F(u)-F(v)+a u-a v\|, \quad \forall v, u \in H, \forall a>0 .
$$

Using inequality (39) with $v=v_{\delta}$ and $u=V_{\delta, \alpha(\delta)}$, Eq. (3) with $a=\alpha(\delta)$, and inequality (35), one gets

$$
\begin{aligned}
\alpha(\delta)\left\|v_{\delta}-V_{\delta, \alpha(\delta)}\right\| & \leq\left\|F\left(v_{\delta}\right)-F\left(V_{\delta, \alpha(\delta)}\right)+\alpha(\delta) v_{\delta}-\alpha(\delta) V_{\delta, \alpha(\delta)}\right\| \\
& =\left\|F\left(v_{\delta}\right)+\alpha(\delta) v_{\delta}-f_{\delta}\right\| \leq \theta \delta .
\end{aligned}
$$

Therefore,

$$
\left\|v_{\delta}-V_{\delta, \alpha(\delta)}\right\| \leq \frac{\theta \delta}{\alpha(\delta)} .
$$

Using (14) and (41), one gets:

$$
\alpha(\delta)\left\|v_{\delta}\right\| \leq \alpha(\delta)\left\|V_{\delta, \alpha(\delta)}\right\|+\alpha(\delta)\left\|v_{\delta}-V_{\delta, \alpha(\delta)}\right\| \leq \theta \delta+\alpha(\delta)\|y\|+\delta .
$$

From the triangle inequality and inequalities (35) and (36) one obtains:

$$
\alpha(\delta)\left\|v_{\delta}\right\| \geq\left\|F\left(v_{\delta}\right)-f_{\delta}\right\|-\left\|F\left(v_{\delta}\right)+\alpha(\delta) v_{\delta}-f_{\delta}\right\| \geq C_{1} \delta^{\gamma}-\theta \delta .
$$


Inequalities (42) and (43) imply

$$
C_{1} \delta^{\gamma}-\theta \delta \leq \theta \delta+\alpha(\delta)\|y\|+\delta .
$$

This inequality and the fact that $C_{1}-\delta^{1-\gamma}-2 \theta \delta^{1-\gamma}>0$ for sufficiently small $\delta$ and $0<\gamma<1$ imply

$$
\frac{\delta}{\alpha(\delta)} \leq \frac{\delta^{1-\gamma}\|y\|}{C_{1}-\delta^{1-\gamma}-2 \theta \delta^{1-\gamma}}, \quad 0<\delta \ll 1 .
$$

Thus, one obtains

$$
\lim _{\delta \rightarrow 0} \frac{\delta}{\alpha(\delta)}=0 .
$$

From the triangle inequality and inequalities (35), (36) and (41) one gets

$$
\begin{aligned}
\alpha(\delta)\left\|V_{\delta, \alpha(\delta)}\right\| & \leq\left\|F\left(v_{\delta}\right)-f_{\delta}\right\|+\left\|F\left(v_{\delta}\right)+\alpha(\delta) v_{\delta}-f_{\delta}\right\|+\alpha(\delta)\left\|v_{\delta}-V_{\delta, \alpha(\delta)}\right\| \\
& \leq C_{2} \delta^{\gamma}+\theta \delta+\theta \delta .
\end{aligned}
$$

This inequality implies

$$
\lim _{\delta \rightarrow 0} \alpha(\delta)\left\|V_{\delta, \alpha(\delta)}\right\|=0 .
$$

The triangle inequality and inequality (12) imply

$$
\begin{aligned}
\alpha\left\|V_{\alpha}\right\| & \leq \alpha\left(\left\|V_{\delta, \alpha}-V_{\alpha}\right\|+\left\|V_{\delta, \alpha}\right\|\right) \\
& \leq \delta+\alpha\left\|V_{\delta, \alpha}\right\| .
\end{aligned}
$$

From formulas (49) and (48), one gets

$$
\lim _{\delta \rightarrow 0} \alpha(\delta)\left\|V_{\alpha(\delta)}\right\|=0 .
$$

It follows from Lemma 2 with $f_{\delta}=f$, i.e., $\delta=0$, that the function $\phi_{0}(a):=a\left\|V_{a}\right\|$ is nonnegative and strictly increasing on $(0, \infty)$. This and relation (50) imply

$$
\lim _{\delta \rightarrow 0} \alpha(\delta)=0 .
$$

From the triangle inequality and inequalities (41) and (12) one obtains

$$
\begin{aligned}
\left\|v_{\delta}-y\right\| & \leq\left\|v_{\delta}-V_{\delta, \alpha(\delta)}\right\|+\left\|V_{\delta, \alpha(\delta)}-V_{\alpha(\delta)}\right\|+\left\|V_{\alpha(\delta)}-y\right\| \\
& \leq \frac{\theta \delta}{\alpha(\delta)}+\frac{\delta}{\alpha(\delta)}+\left\|V_{\alpha(\delta)}-y\right\|,
\end{aligned}
$$

where $V_{\alpha(\delta)}$ solves Eq. (3) with $a=\alpha(\delta)$ and $f_{\delta}=f$.

Conclusion (37) follows from inequalities (46), (51), (52) and Lemma 1. Theorem 7 is proved.

Remark 5. Inequalities (35) and (36) are used as stopping rules for finding approximations:

$$
\alpha(\delta) \approx a(\delta), \text { and } v(\delta) \approx V_{\delta, a(\delta)} .
$$

Remark 6. By the monotonicity of $F$ one gets

$$
\begin{aligned}
\|F(u)-F(v)\|^{2} & \leq\langle F(u)-F(v), F(u)-F(v)+a(u-v)\rangle \\
& \leq\|F(u)-F(v)\|\|F(u)-F(v)+a(u-v)\|, \quad \forall u, v \in H, \forall a>0 .
\end{aligned}
$$

This implies

$$
\|F(u)-F(v)\| \leq\|F(u)-F(v)+a(u-v)\|, \quad \forall u, v \in H, a>0 .
$$

Fix $\delta>0$ and $\theta>0$. Let $C$ be as in Theorem 5. Choose $C_{1}$ and $C_{2}$ such that

$$
C_{1} \delta^{\gamma}+\theta \delta<C \delta^{\gamma}<C_{2} \delta^{\gamma}-\theta \delta
$$

Suppose $\alpha_{i}$ and $v_{i}, i=1,2$, satisfy condition (35) and

$$
\left\|F\left(v_{1}\right)-f_{\delta}\right\|<C_{1} \delta^{\gamma}, \quad C_{2} \delta^{\gamma}<\left\|F\left(v_{2}\right)-f_{\delta}\right\| .
$$


Let us show that

$$
\alpha_{\text {low }}:=\alpha_{1}<a(\delta)<\alpha_{2}:=\alpha_{\text {up }},
$$

where $a(\delta)$ satisfies conditions of Theorem 5 . Using inequality (53) for $v_{i}$ and $V_{\delta, \alpha_{i}}, i=1,2$, and inequality (35), one gets

$$
\begin{aligned}
\left\|F\left(v_{i}\right)-F\left(V_{\delta, \alpha_{i}}\right)\right\| & \leq\left\|F\left(v_{i}\right)-F\left(V_{\delta, \alpha_{i}}\right)+\alpha_{i} v_{i}-\alpha_{i} V_{\delta, \alpha_{i}}\right\| \\
& \leq\left\|F\left(v_{i}\right)+\alpha_{i} v_{i}-f_{\delta}\right\| \leq \theta \delta .
\end{aligned}
$$

From inequalities (55), (57) and the triangle inequality, one derives:

$$
\left\|F\left(V_{\delta, \alpha_{1}}\right)-f_{\delta}\right\|<C_{1} \delta^{\gamma}+\theta \delta \text { and } C_{2} \delta^{\gamma}-\theta \delta<\left\|F\left(V_{\delta, \alpha_{2}}\right)-f_{\delta}\right\| .
$$

Recall that $\left\|F\left(V_{\delta, a(\delta)}\right)-f_{\delta}\right\|=C \delta^{\gamma}$. Inequality (56) is obtained from inequalities (54), (58) and the fact that the function $\phi(\alpha)=\left\|F\left(V_{\delta, \alpha}\right)-f_{\delta}\right\|$ is strictly increasing (see Lemma 2 ).

Let $f_{\delta}, F, C, \theta, \gamma$, and $\delta$ be as in Theorems 5 and 7, and $C_{1}$ and $C_{2}$ satisfy inequality (54). Let us formulate an algorithm (see Algorithm 1 below) for finding $\alpha(\delta) \approx a(\delta)$ and $v(\delta) \approx V_{\delta, a(\delta)}$, using the bisection method and assuming that $F$ is a locally Lipschitz monotone operator and $\alpha_{\text {low }}$ and $\alpha_{u p}$ are known. By Theorem 7, $v(\delta)$ can be considered as a stable solution to Eq. (1).

Algorithm 1. Finding $\alpha(\delta) \approx a(\delta)$ and $v_{\delta} \approx V_{\delta, a(\delta)}$ given $\alpha_{\text {low }}$ and $\alpha_{\text {up }}$.

1. Let $a:=\frac{\alpha_{u p}+\alpha_{\text {low }}}{2}$ and $u_{0}$ be an initial guess for $V_{\delta, a}$. Compute $u_{n}$ by formula (32) and stop at $n_{\text {stop }}$, where $n_{\text {stop }}$ is the smallest $n>0$ for which condition (35) is satisfied. Then go to step 2.

2. If $C_{2} \delta^{\gamma}<\left\|F\left(u_{n_{s t o p}}\right)-f_{\delta}\right\|$, then set $\alpha_{u p}:=a$ and go to step 4. Otherwise, go to step 3 .

3. If $C_{1} \delta^{\gamma} \leq\left\|F\left(u_{n_{\text {stop }}}\right)-f_{\delta}\right\|$, then stop the process and take $v(\delta):=u_{n_{\text {stop }}}$ as a solution to (1). If $\left\|F\left(u_{n_{\text {stop }}}\right)-f_{\delta}\right\|<C_{1} \delta^{\gamma}$, then set $\alpha_{\text {low }}:=a$ and go to step 4

4. Check if $\left\|a-\alpha_{\text {low }}\right\|$ is less than a desirable small value $\epsilon>0$. If it is, then take $v(\delta):=u_{n_{\text {stop }}}$ as a solution to (1). If is is not, then go back to step 1 .

Let us formulate algorithms for finding $\alpha_{u p}$ and $\alpha_{\text {low }}$.

Algorithm 2. Finding $\alpha_{\text {up }}$.

1. Let $a=\alpha$ be an initial guess for $\alpha(\delta)$ and $u_{0}$ be an initial guess for $v_{\delta}$. Compute $u_{n}$ by formula (32) with $a$ and stop at $n_{\text {stop }}$, the smallest $n>0$ for which condition (35) is satisfied. Then go to step 2 .

2. If condition (36) holds for $v_{\delta}:=u_{n_{s t o p}}$, then stop the process and take $u_{n_{\text {stop }}}$ as a solution to (1). Otherwise, go to step 3.

3. If $C_{2} \delta^{\gamma}<\left\|F\left(u_{n_{s t o p}}\right)-f_{\delta}\right\|$, then set $\alpha_{u p}:=a$. Otherwise, set $\alpha:=2 a$ and go back to step 1 .

Algorithm 3. Finding $\alpha_{\text {low }}$.

1. Let $a=\alpha$ be an initial guess for $\alpha(\delta)$ and $u_{0}$ be an initial guess for $v_{\delta}$. Compute $u_{n}$ by formula (32) with $a$ and stop at $n_{\text {stop }}$, the smallest $n>0$ for which condition (35) is satisfied. Then go to step 2 .

2. If condition (36) holds for $v_{\delta}:=u_{n_{\text {stop }}}$, then stop the process and take $u_{n_{\text {stop }}}$ as a solution to (1). Otherwise, go to step 3 .

3. If $\left\|F\left(u_{n_{\text {stop }}}\right)-f_{\delta}\right\|<C_{1} \delta^{\gamma}$, then set $\alpha_{\text {low }}:=a$. Otherwise, set $\alpha:=\frac{a}{2}$ and go back step 1 .

In practice these algorithms are often implemented at the same time to avoid repetition calculations.

Remark 7. The sequence $\left(\left\|u_{n}-V_{\delta, a(\delta)}\right\|\right)_{n=0}^{\infty}$, where $u_{n}$ is computed by formula (32) and $V_{\delta, a(\delta)}$ is the solution to (3) with $a=a(\delta)$, is decreasing. Thus, the sequence $u_{n}$ will stay inside a ball $B(0, R)$ assuming that $R>0$ is chosen sufficiently large, so that $y, u_{0} \in B(0, R)$.

Remark 8. Theorem 7 and the above algorithms are not only useful for solving nonlinear equations with monotone operators but also for solving linear equations with monotone operators. If one uses iterative methods to solve Eq. (29) then, by using Theorem 7, one can stop iterations whenever inequality (35) holds. By using stopping rule (35) one saves time of computation compared to solving (29) exactly. If $F$ is a positive matrix then one can solve (29) by conjugate gradient, or Jacobi, or Gauss-Seidel, or successive over-relaxation methods, with stopping rule (35).

Remark 9. If $F$ is twice Fréchet differentiable, there are more options for solving Eqs. (3) and (33): they can be solved by gradient-type methods, Newton-type methods, or a combination of these methods. 


\section{References}

[1] K. Deimling, Nonlinear Functional Analysis, Springer Verlag, Berlin, 1985.

[2] J.L. Lions, Quelques methodes de resolution des problemes aux limites non lineaires, Dunod, Gauthier-Villars, Paris, 1969.

[3] D. Pascali, S. Sburlan, Nonlinear Mappings of Monotone Type, Noordhoff, Leyden, 1978.

[4] A.G. Ramm, Theory and Applications of Some New Classes of Integral Equations, Springer-Verlag, New York, 1980.

[5] A.G. Ramm, Stationary regimes in passive nonlinear networks, in: P. Uslenghi (Ed.), Nonlinear Electromagnetics, Acad. Press, New York, 1980, pp. 263-302.

[6] A.G. Ramm, Iterative solution of linear equations with unbounded operators, J. Math. Anal. Appl. 1338-1346.

[7] A.G. Ramm, On unbounded operators and applications, Appl. Math. Lett. 21 (2008) 377-382.

[8] A.G. Ramm, Inverse Problems, Springer, New York, 2005.

[9] A.G. Ramm, Dynamical Systems Method for Solving Operator Equations, Elsevier, Amsterdam, 2007.

[10] I.V. Skrypnik, Methods for Analysis of Nonlinear Elliptic Boundary Value Problems, American Mathematical Society, Providence, RI, 1994.

[11] U. Tautenhahn, On the method of Lavrentiev regularization for nonlinear ill-posed problems, Inverse Problems 18 (2002) $191-207$.

[12] M.M. Vainberg, Variational Methods and Method of Monotone Operators in the Theory of Nonlinear Equations, Wiley, London, 1973. 